\title{
THE RELATIONSHIP BETWEEN STEM VESSEL PARAMETERS AND THE DEVELOPMENT OF STRATA IN THE EARLY STAGES OF SECONDARY FOREST SUCCESSION IN AMAZONIA
}

\author{
Akio TSUCHIYA ${ }^{1}$, Yoshihiko, HIRABUKI ${ }^{2}$, Toshie NISHIZAWA ${ }^{3}$, \\ Pedro Braga LISBOA ${ }^{4}$, Carlos Rosario da SILVA ${ }^{4}$
}

ABSTRACT - In order to compare the development of strata in the early stages of secondary forest succession with vessel parameters of the tree species, a forest inventory was carried out in 4-year (Q1: $\left.48 \mathrm{~m}^{2}\right)$, 11-year (Q2: $\left.400 \mathrm{~m}^{2}\right)$ and 20-year (Q3: $\left.400 \mathrm{~m}^{2}\right)$ forests and vessel parameters were investigated from stem cross sections of 18 species obtained in Q2. Thirty three species (21 families), 77 species (35 families), 39 species (20 families) were found in Q1, Q2, Q3, respectively. The percentage of dead individuals, dead stems and the percentage of individuals with multiple stems increased with time after clear cutting. Also, the total $\mathrm{D}^{2} \mathrm{H}$ of Q3 was 26.1 times that of Q1, and the development of strata started in Q2 and Q3. The image analysis of vessel size, area and number of vessels revealed that species which reach the forest canopy had a large $D^{2} \mathrm{H}$ value, vessel diameter and area, while species which remain near the forest floor had smaller ones. Poecilanthe effusa (Huber) Ducke is an example of the latter case, with a large number of individuals and abundant sprouting of new stems from stumps, but with high mortality.

Key-words: Amazonia, secondary forest, strata, image analysis, vessels

\section{A Relação entre os Parâmetros de Vasos no Tronco e Desenvolvimento de Estratos nos Estágios Iniciais de Sucessão em Florestas Secundárias na Amazônia}

RESUMO - A fim de comparar o desenvolvimento dos estratos no estágio inicial da floresta secundária no Amazônia com os parâmetros de vasos nas espécies de árvores, um inventário florestal foi elaborado em florestas de 4-anos (Q1: $\left.48 \mathrm{~m}^{2}\right), 11$-anos (Q2: $\left.400 \mathrm{~m}^{2}\right)$ e 20-anos (Q3: $400 \mathrm{~m}^{2}$ ) e os parâmetros de vasos foram investigados através dos cortes transversais dos troncos em 18 espécies em Q2. Apesar de serem encontradas 33 espécies (21 familias), 77 espécies (35 familias), 39 espécies (20 familias) nas respectivas áreas Q1, Q2 e Q3, a porcentagem de mortes individuais, troncos mortos e a porcentagem de caules individuais em relação a caules germinados aumentou com a passagem do tempo após o período de desmatamento. Também, foi observado que, o $\mathrm{D}^{2} \mathrm{H}$ total no Q3 foi 26.1 vezes maior que em Q1 e o desenvolvimento dos estratos iniciaram-se em Q2 e Q3. As análises de imagens do tamanho do vaso, área celular e do número de vasos, demonstraram que as espécies que poderiam chegar a compor a cobertura florestal, apresentam grandes valores em $\mathrm{D}^{2} \mathrm{H}$, diametro de vasos e área. Enquanto as espécies que poderiam permanencer no sub-bosque demonstraram baixos valores dos mesmos parâmetros. Poecilanthe effusa (Huber) Ducke e um exemplo do segundo caso: possue grande número de indíviduos e germina consideravelmente um bom número de novos caules, mas apresenta grande taxa de mortalidade.

Palavras-chave: Amazônia, floresta secundária, estratos, análises imagens, vasos

'Faculty of Integrated Arts and Sciences, Hiroshima University, 1-7-1 Kagamiyama, HigashiHiroshima, Hiroshima 739-8521, Japan

${ }^{2}$ Faculty of Education, Miyagi University of Education, Aoba, Aramaki, Aoba-ku, Sendai, Miyagi 980-8578, Japan

${ }^{3}$ Faculty of Humanities, Tokyo Seitoku University, 2014 Nakadaiyatsu, Hoshina, Yachiyo, Chiba 276-0013, Japan

${ }^{4}$ Department of Botany, Museu Paraense Emílio Goeldi, 376 Av. Magalhães Barata, Belém PA 66040-170, Brazil 


\section{Introduction}

In a natural tropical rain forest, the forest structure is usually divided into three layers with the passage of time, and each space is occupied by trees adapted to the light conditions in that layer (Federov, 1966). If a gap is formed in the forest for any reason, however, competition among the trees established in the gap starts for space (Platt \& Strong, 1989, Vazques-Yanes \& Orozco-Segovia, 1994). Competition generated by natural causes, such as fallen trees and heavy rains, is widely studied, but recently huge artificial clear-cuts have been examined also (Fearnside, 1990, 1991).

In the past, many studies of Amazonian tropical humid forests have focused on exploitation and evaluation of timber resources. Project RADAMBRASIL is a typical example. Remote sensing of forest/mineral resources was carried out in the whole Brazilian Amazon, and fieldwork on species composition and aboveground biomass was also conducted by the Instituto Nacional de Pesquisas da Amazônia - INPA, Museu Paraense Emílio Goeldi - MPEG and Empresa Brasileira de Pesquisa Agropecuária EMBRAPA. Also, wood anatomical characteristics of useful species were investigated in the laboratory (SUDAM/ DRN, 1981, Loureiro et al., 1997). These projects described in detail the geographical distribution of commercially valuable species, biodiversity and wood biomass. However, they were intended for natural forests. During the 1980 s, interest changed toward the expansion of pasture/roads and the disap- pearance of forested areas. Further, experiments to minimize disturbance, simulation studies of logging interval to maximize yield, and afforestation programs began. However, there are few studies that investigate natural vegetation recovery in early plant succession (Carvalho, 1986, Taylor et al., 1995), and there is no study on the relationship between secondary forests with low commercial value and wood quality.

Aside from light intensity, water supply is also indispensable for the growth of trees. The parameters of vessels in which sap flow passes from the root are believed to be a key to understanding the growth pattern of trees (Shimaji et al., 1976, Schweingrüber, 1988). Therefore, we investigated species composition in three secondary forests with different ages, and investigated the relationship between wood biomass and vessel parameters to try to elucidate details of competition for space among species occurring in the early stages of secondary forest succession.

\section{Material and Methods}

\section{Fieldwork}

The study area was located in the Ferreira Penna Scientific Station (Lat.: $1^{\circ} 42^{\prime} 30^{\prime \prime S}$, Long.: 51'31'45"W, Area: $33,100 \mathrm{ha}$ ) managed by MPEG. This station is part of Caxiuanã National Forest, approximately $400 \mathrm{~km}$ from Belém, Pará. Annual precipitation is $2,500 \mathrm{~mm}$ in Breves and $1,930 \mathrm{~mm}$ in Altamira, annual mean temperature is $26^{\circ} \mathrm{C}$, but the climate type is categorized as Am (tropical monsoon climate) because of a weak dry season during July to December (SUDAM/ 
PHCA, 1984). The terrain has a gentle slope, and the ground surface is covered with a shallow medium yellow latosol originating from mid-Tertiary sediments (Gatto, 1991).

Fieldwork was carried out in July and August, 1995. The general area has natural forests with no disturbance, but there are some areas where the forest was clear cut to construct helicopter and Cessna bases that were abandoned without being used (De La Penha et al., 1990). Quadrats were established in secondary forests whose history was known. They were 4-year, 11-year and 20-year forests, respectively (hereafter, called Q1, Q2 and $\mathrm{Q} 3$, respectively). The area of Q1 was $48 \mathrm{~m}^{2}$, and the latter two quadrats were $400 \mathrm{~m}^{2}$. In the forest inventory, tree species were identified, individual trees ( $>1.3 \mathrm{~m}$ in height) were mapped within the quadrat, stem and lowest leaf layer heights were measured with a telescoping pole, basal diameter (Q1) and DBH (Q2, Q3) were measured with a vernier caliper/measure tape. The status of stems (living/dead, geminate from seeds/regenerated from stumps) and the number of live and dead stems were also investigated. When a tree had multiple stems, the height and DBH of the tallest stem were measured. Palms were also included in the inventory. For palms, the highest leaf layer was regarded as the plant height, and the diameter at the rosette-like basal portion was substituted for the DBH. The $\mathrm{D}^{2} \mathrm{H}$, an index of stem biomass, was calculated from the product of a square of DBH and tree height.

\section{Tree Disk Sampling and Image Analysis}

Tree disks were obtained by cutting down the stems at $30 \mathrm{~cm}$ to $1 \mathrm{~m}$ above the ground. The number of disks was 11 in Q1, 39 in Q2 and 6 in Q3 (families: 20, species: 29). For species with two or more disks, care was taken to obtain tree disks having as different a stem size as possible, although this was impossible for every species. The disks were brought back to laboratory after painting with acrylic resin (Asahi, A-011) to stop mold. In addition, 39 samples of 18 species in 12 families obtained in Q2 were investigated in order to discuss the species differences and the relations to biomass in a same aged forest. The species are: Annona montana Macf., Annona paludosa Aubl,, Byrsonima crispa ADR. Juss., Bowdichia nitida ex Benth., Cochlospermum orinocensis (H.B.K.) Steud., Cupania hispida SW, Croton matourensis Aubl., Cupania rubiginosa (Poir.) Radlk., Dipterix odorata (Aubl.) Willd., Enterolobium schomburghii Benth., Hirtella triandra SW., Lacistema polystachum Schniz., Myrcia fallax (Rich.) DC., Maprounea guianensis Aubl., Poecilanthe effusa (Huber) Ducke, Sclerolobium paniculatum Vog., Sclerolobium paraense Huber, Vismia cayenensis (Jacq.) Pers.

The disks were polished with a grinder (Nichika, RG-5), to which sand paper (\#150, \#400) was attached. Then, they were processed into chips of $1 \mathrm{~mm}$ thickness using a chip saw (Makita, 255). Next, a chip was glued 
to a slide glass (Konishi, Bond E Set), and was polished by a grinder, using fine sand paper ( $\# 800, \# 2,000$ ). The reason a microtome was not used is that this requires small sections and is not appropriate for large specimens. Tree disks of over $20 \mathrm{~cm}$ in diameter were divided into smaller pieces because the size was too large to slice by chip saw.

The image analysis system used in this study takes in an image through a CCD camera (Tokyo Electronic Industry, CS-5510) from a measure scope (Nikon, MM-22). The reflected images of chip samples were converted into digital signals by an analog capture board (Neotech, IG-24$\mathrm{PCI}$ ) and were inputted into a computer. The data was processed by image analysis software (Mitani, MacScope 2.5). Length and brightness of the input image were calibrated, and ROI (range of interest) was set up. The image was transformed into black \& white, and then the object (vessels) was extracted. It is difficult to distinguish vessels from axial and ray parenchyma because they sometimes have the same whitish brightness. In this case, the location of vessels on the monitor was marked with a manually operated pen. Finally, the area, diameter, circumference of each vessel were measured. The average, total and standard deviation of these parameters were calculated. These series of analyses were conducted four times for a tree-ring from 1994, the year prior to the fieldwork for the obtained samples. When a tree-ring width of one year was too large to input at a time, it was subdivided into several images.

\section{Results}

\section{Species Composition and Forest Structure}

The number of species in Q1 was 33 with 165 live and 22 dead individuals, 246 live stems and 24 dead stems (Tab. 1). The percentage of individuals sprouted from stumps was $28.0 \%$ and the mean number of stems per stump was $7.2 \pm 81$. The species with more than 10 live individuals were $P$. guianensis $(\mathrm{n}=34), V$. guianensis (32), O. longifolia (28) and $M$. fallax (16). These 4 species comprised $60.6 \%$ of all individuals. The number of tree species appearing in Q2 was 75, and two palm species were also found. There were 540 live and 46 dead individuals, 904 live stems and 55 dead stems. The percentage of individuals with multiple stems was $29.0 \%$ and the mean stem number was $3.6 \pm 1.9$. In this quadrat, it could not be determined whether the individual had germinated from seed or sprouted from a dead stem because the original stumps (if any) had decomposed. The species with over 20 live individuals were $P$. effusa $(\mathrm{n}=129)$, C. rubiginosa (74), C. hispida (27), and these 3 species accounted for $42.6 \%$ of all individuals. In Q3, 35 tree species and 4 palm species appeared. There were 225 live and 67 dead individuals, 456 live stems and 128 dead stems. Both live individuals and stems decreased in number, while dead ones increased in comparison with Q2. Also, the percentage of individuals with multiple 
Table 1. Number of live individuals, stems, dead individuals and stems (>1.3 m) in 4-year (Q1: 48 $\mathrm{m}^{2}$ ), 11-year and 20-year forests (Q2 \& Q3: $400 \mathrm{~m}^{2}$ ) in the Caxiuanã National Forest, Pará, Brasil.

\begin{tabular}{|c|c|c|c|c|c|c|c|c|c|c|c|c|c|c|}
\hline \multirow{3}{*}{ No. } & \multirow{3}{*}{ Species } & \multirow{3}{*}{ Family } & \multicolumn{4}{|c|}{ Q1 (48 m2) } & \multicolumn{4}{|c|}{ Q2 (400 m2) } & \multicolumn{4}{|c|}{ Q3 (400 m2) } \\
\hline & & & \multicolumn{2}{|c|}{ Live } & \multirow[b]{2}{*}{ Ind. } & \multirow{2}{*}{$\begin{array}{l}\text { Dead } \\
\text { Stem } \\
\end{array}$} & \multicolumn{2}{|c|}{ Live } & \multirow{2}{*}{\multicolumn{2}{|c|}{$\begin{array}{r}\text { Dead } \\
\text { Ind. Stem } \\
\end{array}$}} & \multicolumn{2}{|c|}{ Live } & \multicolumn{2}{|c|}{ Dead } \\
\hline & & & Ind. & Stem & & & Ind. & Stem & & & Ind. & Stem & Ind. & Stem \\
\hline 1 & Aegiphila amazonica & VERB & 2 & 2 & . & . & 1 & 1 & . & - & $\therefore$ & . & - & - \\
\hline 2 & Alibertia edulis & RUBI & 1 & 6 & . & - & . & - & - & - & - & . & - & - \\
\hline 3 & Ambelania acida & APOC & - & $\cdot$ & - & . & 3 & 3 & . & , & - & . & - & - \\
\hline 4 & Annona montana & ANNO & - & - & - & - & 2 & 3 & - & - & . & : & - & - \\
\hline 5 & Annona paludosa & ANNO & - & - & - & , & 4 & 4 & 2 & 2 & - & . & - & ? \\
\hline 6 & Aspidosperma desmanthum & APOC & - & . & - & - & 3 & 3 & - & - & - & . & . & . \\
\hline 7 & Astrocaryum mumbaca & PALM & - & . & - & . & 3 & 5 & - & - & 7 & 7 & 4 & 4 \\
\hline 8 & Attalea cf. spectabilis & PALM & - & $\cdot$ & - & - & & + & . & - & 52 & 52 & - & - \\
\hline 9 & Barana guianensis & FLAC & 1 & 1 & , & . & - & - & - & - & - & - & - & . \\
\hline 10 & Bowdichia nitida & L.PAP & - & - & , & - & . & $=$ & - & - & 1 & 2 & . & \\
\hline 11 & Brosium guianensis & MORA & - & - & - & - & 4 & 7 & - & - & - & - & - & . \\
\hline 12 & Byrsonima crispa & MALP & - & $\cdot$ & . & - & 1 & 1 & - & - & 1 & 1 & - & - \\
\hline 13 & Calliandra surinamensis & L-MIM & 3 & 6 & 1 & 1 & . & . & - & . & $=$ & . & - & - \\
\hline 14 & Caryocar glabrum & CARY & - & - & . & . & 1 & 1 & - & - & - & . & . & - \\
\hline 15 & Casearia arborea & FLAC & - & - & - & - & 1 & 1 & $=$ & , & . & - & : & . \\
\hline 16 & Casearia decandra & FLAC & 2 & 2 & - & 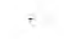 & 2 & 2 & 1 & 1 & . & - & - & - \\
\hline 17 & Casearia javitensis & FLAC & - & - & . & • & 14 & 29 & 1 & 1 & 1 & 2 & 1 & 2 \\
\hline 18 & Casearia ulmifolia & FLAC & - & . & . & - & 4 & 5 & 2 & 2 & . & - & . & . \\
\hline 19 & Cecropia palmata & MORA & 3 & 3 & - & . & - & - & - & . & - & . & - & . \\
\hline 20 & Clarisia ilicifolia & MORA & . & $\therefore$ & - & - & 2 & 2 & - & . & - & - & - & . \\
\hline 21 & Cochlospermum orinocensis & $\mathrm{COCH}$ & 1 & 1 & - & - & 12 & 12 & 4 & 5 & - & - & - & - \\
\hline 22 & Cordia exaltata & BORA & - & . & - & - & 3 & 6 & - & . & 4 & 6 & 2 & 2 \\
\hline 23 & Couma guianensis & APOC & 1 & 2 & . & $;$ & - & . & . & - & - & . & - & - \\
\hline 24 & Couratari cf. tauari & LECY & - & - & $=$ & $\cdot$ & 1 & 4 & - & - & - & . & - & , \\
\hline 25 & Crepidospermum gondotianum & BURS & . & - & - & - & 1 & 1 & - & - & - & $=$ & - & . \\
\hline 26 & Croton matourensis & EUPH & $\cdot$ & . & - & - & 12 & 14 & 1 & 1 & - & - & - & - \\
\hline 27 & Cupania diphylla & SAPI & - & - & : & - & 6 & 6 & - & - & - & - & - & - \\
\hline 28 & Cupania hispida & SAPI & - & . & - & . & 27 & 46 & 2 & 3 & 2 & 2 & 1 & 3 \\
\hline 29 & Cupania rubiginosa & SAPI & - & $\therefore$ & - & . & 74 & 108 & 3 & 4 & 1 & 1 & - & * \\
\hline 30 & Cupania scrobiculata & SAPI & 2 & 6 & 1 & 1 & - & - & - & - & - & - & - & . \\
\hline 31 & Didymopanax morototoni & ARAL & - & $\because$ & . & . & 1 & 1 & - & - & - & . & - & - \\
\hline 32 & Dipterix odorata & L.PAP & . & . & - & - & 3 & 5 & . & . & - & . & . & . \\
\hline
\end{tabular}


Table 1. Cont.

\begin{tabular}{|c|c|c|c|c|c|c|c|c|c|c|c|c|c|c|}
\hline \multirow{3}{*}{ No. } & \multirow{3}{*}{ Species } & \multirow{3}{*}{ Family } & \multicolumn{4}{|c|}{ Q1 (48 m2) } & \multicolumn{4}{|c|}{ Q2 (400 m2) } & \multicolumn{4}{|c|}{ Q3 $(400 \mathrm{~m} 2)$} \\
\hline & & & \multicolumn{2}{|c|}{ Live } & \multicolumn{2}{|c|}{ Dead } & \multicolumn{2}{|c|}{ Live } & \multicolumn{2}{|c|}{ Dead } & \multicolumn{2}{|c|}{ Live } & \multicolumn{2}{|r|}{ Dead } \\
\hline & & & Ind. & Stem & Ind. & Stem & Ind. & Stem & Ind. & Stem & Ind. & Stem & Ind. & Stem \\
\hline 33 & Duguetia iongicuspis & ANNO & $\cdot$ & $\cdot$ & $\cdot$ & - & 1 & 2 & $\cdot$ & $\cdot$ & - & + & - & - \\
\hline 34 & Enterolobium schomburghii & L-MIM & - & $\cdot$ & , & + & 1 & 1 & - & . & . & - & , & - \\
\hline 35 & Eschweilera ct. bracteata & LECY & $\cdot$ & $\cdot$ & - & $\cdot$ & - & -. & $\cdot$ & . & 1 & 1 & . & - \\
\hline 36 & Eschweilera coriacea & LECY & $\cdot$ & - & $\cdot$ & - & 1 & 4 & $\cdot$ & $=$ & - & $\cdot$ & 3 & - \\
\hline 37 & Eugenia cf. bifilora & MYRT & - & - & • & - & - & $\cdot$ & - & - & 1 & 2 & 1 & 1 \\
\hline 38 & Eugenia egensis & MYRT & - & $\cdot$ & . & $\cdot$ & $\cdot$ & - & - & - & 3 & 6 & · & - \\
\hline 39 & Eugenia of. omissa & MYRT & 1 & 1 & $\tau$ & - & , & $*$ & - & - & $=$ & $\cdot$ & : & - \\
\hline 40 & Eugenia patrisii & MYRT & 1 & 5 & . & $\cdot$ & 8 & 13 & , & $\cdot$ & 1 & 1 & - & $\cdot$ \\
\hline 41 & Faramea anisocalyx & RUBI & 3 & 9 & $\cdot$ & $\cdot$ & • & • & $\cdot$ & $\cdot$ & • & $\cdot$ & *. & $\cdot$ \\
\hline 42 & Geyssospermum cericeum & APOC & - & - & $\cdot$ & - & 4 & 12 & - & - & 1 & 4 & - & $\cdot$ \\
\hline 43 & Goupia glabra & CELA & - & - & - & - & 2 & 2 & $\cdot$ & - & 3 & 4 & $=$ & - \\
\hline 44 & Guatteria poeppigiana & ANNO & - & - & * & - & 2 & 2 & 1 & 2 & * & - & ; & - \\
\hline 45 & Himatanthus scuuba & APOC & - & , & - & $\cdot$ & 3 & 4 & - & - & 1 & 1 & - & - \\
\hline 46 & Hirtella burchellit & CHRY & - & $\cdot$ & $\cdot$ & . & 14 & 25 & 1 & 1 & 23 & 48 & 2 & 2 \\
\hline 47 & Hirtella triandra & CHRY & 1 & 3 & 1 & 2 & 14 & 29 & 1 & 1 & 4 & 11 & 1 & 1 \\
\hline 48 & Hymenolobium llavum & L.PAP & - & $\cdot$ & . & $\cdot$ & 1 & 1 & $\cdot$ & $\cdot$ & - & . & - & - \\
\hline 49 & Inga heterophylla & L-MIM & 3 & 7 & 1 & 1 & $\cdot$ & $\cdot$ & - & $\cdot$ & 2 & 12 & 4 & 5 \\
\hline 50 & Lacistema polystachyum & LACI & - & $\cdot$ & • & - & 8 & 24 & 2 & 2 & 1 & 2 & , & - \\
\hline 51 & Lacunaria minor & QUIN & $\cdot$ & , & - & $\cdot$ & 1 & 1 & - & $\cdot$ & - & - & . & 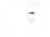 \\
\hline 52 & Lecythis pisonis & LECY & $\cdot$ & $\cdot$ & , & - & • & $\cdot$ & * & · & 4 & 4 & . & $\cdot$ \\
\hline 53 & Licania canescens & CHRY & 2 & 11 & 1 & 2 & 2 & 3 & $=$ & . & 8 & 8 & 1 & 1 \\
\hline 54 & Licania cf. heteromorpha & CHRY & $\cdot$ & $\cdot$ & $\cdot$ & . & 2 & 2 & - & . & $\cdot$ & , & * & $\cdot$ \\
\hline 55 & Lindackeria pauciflora & FLAC & 1 & 4 & $\cdot$ & $\cdot$ & $\cdot$ & . & $\cdot$ & . & $\cdot$ & . & · & $\cdot$ \\
\hline 56 & Maprounea guianensis & EUPH & 6 & 7 & • & - & 10 & 14 & $\cdot$ & - & 5 & 13 & 5 & 8 \\
\hline 57 & Matayba cf. arborescens & SAPI & • & $\cdot$ & . & - & 1 & 1 & - & - & - & $\cdot$ & : & - \\
\hline 58 & Maximiliana maripa & PALM & - & - & - & - & 6 & 6 & - & - & 2 & 2 & - & - \\
\hline 59 & Miconia eriodonta & MELA & 1 & 10 & 1 & 1 & $\cdot$ & $\cdot$ & $\cdot$ & - & 2 & 2 & $\cdot$ & $\cdot$ \\
\hline 60 & Miconia poeppigiana & MELA & - & - & - & $\cdot$ & 1 & 1 & $=$ & - & - & $\cdot$ & $\cdot$ & - \\
\hline 61 & Miconia splendens & MELA & - & $\cdot$ & $\cdot$ & $\cdot$ & 8 & 8 & - & $*$ & - & $\cdot$ & $\cdot$ & $\cdot$ \\
\hline 62 & Micropholis venulosa & SAPO & - & $\cdot$ & - & $\cdot$ & 1 & 1 & - & $\cdot$ & - & $\cdot$ & - & - \\
\hline 63 & Morinda triphylla & RUBI & - & - & $\cdot$ & $\cdot$ & 1 & 1 & $\cdot$ & . & - & - & - & - \\
\hline 64 & Myrcia bracteata & MYRT & 2 & 4 & 1 & 1 & 16 & 16 & $\cdot$ & r & $\cdot$ & $\cdot$ & - & $\cdot$ \\
\hline 65 & Myrcia fallax & MYRT & 16 & 22 & 1 & 1 & 8 & 9 & 1 & 1 & - & $\cdot$ & - & - \\
\hline 66 & Myrcia sylvatica & MYRT & 1 & 2 & $\cdot$ & - & 1 & 1 & $\cdot$ & - & $\cdot$ & . & , & - \\
\hline 67 & Neer $s p$. & NICT & 2 & 4 & 2 & 2 & 7 & 11 & $\cdot$ & $\cdot$ & 1 & 1 & - & , \\
\hline 68 & Ocotea longitolia & LAUR & 28 & 34 & 1 & 1 & - & $\cdot$ & - & $\cdot$ & - & - & . & - \\
\hline 69 & Oenocarpus distichus & PALM & - & - & $\cdot$ & - & - & $\cdot$ & $\cdot$ & ? & 1 & 1 & ? & - \\
\hline 70 & Ormosia flava & L.PAP & $\cdot$ & $\cdot$ & • & $\cdot$ & 2 & 3 & . & $\cdot$ & $\cdot$ & $\cdot$ & $\cdot$ & $\cdot$ \\
\hline
\end{tabular}


Table 1. Cont.

\begin{tabular}{|c|c|c|c|c|c|c|c|c|c|c|c|c|c|c|}
\hline \multirow{3}{*}{ No, } & \multirow{3}{*}{ Species } & \multirow{3}{*}{ Family } & \multicolumn{4}{|c|}{ Q1 (48 m2) } & \multicolumn{4}{|c|}{ Q2 (400 m2) } & \multicolumn{4}{|c|}{$\mathrm{Q} 3(400 \mathrm{~m} 2)$} \\
\hline & & & \multicolumn{2}{|c|}{ Live } & \multicolumn{2}{|c|}{ Dead } & \multicolumn{2}{|c|}{ Liv } & \multicolumn{2}{|c|}{ Dead } & \multicolumn{2}{|c|}{ Live } & \multicolumn{2}{|c|}{ Dead } \\
\hline & & & Ind. & Stem. & Ind. & Stem & Ind. & Stem & Ind. & Stem & Ind. & Stem & Ind. & Stem \\
\hline 71 & Ormosia paraensis & L-PAP & - & - & . & , & - & - & ? & - & 4 & 14 & . & . \\
\hline 72 & Ouratea castanhefolia & OCHN & . & - & . & - & - & - & - & - & 1 & 1 & . & - \\
\hline 73 & Ouratea paraensis & OCHN & . & - & - & - & 1 & 1 & - & $\cdot$ & . & . & . & - \\
\hline 74 & Palicourea guianensis & RUBI & 34 & 34 & 1 & 1 & 2 & 2 & - & $\cdot$ & $\cdot$ & - & . & - \\
\hline 75 & Parkia igeiffora & L-MIM & . & - & . & - & 1 & 1 & - & . & - & - & . & - \\
\hline 76 & Pipthecellobium cf. latifolium & L-MIM & $\cdot$ & - & - & - & 1 & 1 & - & - & $\cdot$ & - & - & - \\
\hline 77 & Piptocarpa opaca & COMP & 5 & 8 & . & - & . & - & - & . & - & - & - & - \\
\hline 78 & Poecilanthe effusa & L.PAP & . & - & - & · & 129 & 326 & 17 & 19 & 39 & 148 & 22 & 65 \\
\hline 79 & Pogonophora schomburgkiana & EUPH & - & - & - & - & - & - & - & - & 29 & 78 & 11 & 15 \\
\hline 80 & Protium decandrum & BURS & - & - & - & . & 1 & 1 & - & . & $\cdot$ & - & . & - \\
\hline 81 & Protium guianense & BURS & - & - & - & - & 6 & 6 & . & - & . & r & - & . \\
\hline 82 & Protium pilosum & BURS & - & - & - & . & 1 & 1 & - & - & : & . & . & + \\
\hline 83 & Protium trifoliolatum & BURS & * & * & - & . & 1 & 1 & , & - & , & - & - & - \\
\hline 84 & Psidium acutangulum & MYRT & 1 & 3 & 1 & 1 & . & * & . & - & . & - & - & . \\
\hline 85 & Pterocarpus rohrii & L.PAP & . & $\cdot$ & $\cdot$ & . & 1 & 1 & - & - & . & $\because$ & · & . \\
\hline 86 & Rhabdodendron amazonicum & RUTA & $\cdot$ & . & ; & . & 2 & 2 & , & - & . & . & . & . \\
\hline 87 & Rollinia exucca & ANNO & 2 & 2 & . & - & . & . & . & . & . & . & : & . \\
\hline 88 & Ryania pyrifera & FLAC & $\cdot$ & . & - & - & 17 & 19 & - & - & . & $\cdot$ & - & . \\
\hline 89 & Sclerolobium melanocarpon & L.CAE & - & . & . & . & 7 & 7 & . & - & - & - & - & : \\
\hline 90 & Sclerolobium paniculatum & L.CAE & 1 & 1 & . & - & 5 & 5 & - & - & 1 & 1 & · & · \\
\hline 91 & Scierolobium paraense & L-CAE & $\cdot$ & - & - & - & 5 & 5 & . & . & : & . & . & - \\
\hline 92 & Simaba cedron & SIMA & $\cdot$ & - & - & . & 1 & 1 & - & . & . & $\cdot$ & . & - \\
\hline 93 & Siparuna guianensis & MONI & 1 & 1 & $\cdot$ & - & 2 & 2 & - & . & . & 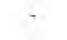 & $\cdot$ & $\cdot$ \\
\hline 94 & Solano leucocarpon & SOLA & 2 & 2 & $\cdot$ & - & - & - & , & . & $\cdot$ & . & - & - \\
\hline 95 & Sorocea steimbachii & MORA & - & . & . & . & 1 & 1 & . &. & - & . & . & . \\
\hline 96 & Stryphnodendron pulcherrimum & L-MIM & 2 & 7 & 1 & 1 & - & . & - & - & 1 & 1 & - & - \\
\hline 97 & Swartzia brachyrachis & L-MIM & . & - & - & - & 3 & 9 & 1 & 2 & 3 & 3 & - & $\cdot$ \\
\hline 98 & Swartzia cardiosperma & L.CAE & - & - & - & - & • & $\cdot$ & . & . & 1 & 1 & - & . \\
\hline 99 & Swartzia racemosa & L.CAE & - & - & - & - & - & . & . & . & 1 & 1 & . & - \\
\hline 100 & Talisia Iongifolia & SAPI & • & $\cdot$ & - & $\cdot$ & - & · & . & - & 2 & 2 & - & $\cdot$ \\
\hline 101 & Talisia mollis & SAPI & . & $\cdot$ & - & - & 6 & 6 & · & - & 3 & 3 & - & - \\
\hline 102 & Tapirira guianensis & ANAC & . & : & . & - & 5 & 5 & - & , & 1 & 1 & - & . \\
\hline 103 & Tetragastris panamensis & BURS & · & · & . & - & 2 & 2 & . & . & - & - & - & - \\
\hline 104 & Theobroma speciosum & STER & . & . & - & . & 2 & 4 & 1 & 3 & - & - & . & - \\
\hline 105 & Trattinickia rhoifolia & BUAS & . & $\cdot$ & . & : & 19 & 20 & - & $=$ & . & . & . & . \\
\hline 106 & Trema micrantha & ULMA & 1 & 1 & . & $\cdot$ & . & - & . & - & . & - & . & $\cdot$ \\
\hline 107 & Vismia cayenensis & GUTT & - & - & . & - & 4 & 4 & . & - & . & - & . & - \\
\hline 108 & Vismia guianensis & GUTT & 32 & 35 & . & - & - & . & . & : & - & - & . & ? \\
\hline 109 & Vochysia densiffora & $\mathrm{VOCH}$ & - & - & - & - & 4 & 4 & - & . & . & . & . & - \\
\hline \multirow[t]{3}{*}{110} & Xylopia amazonica & ANNO & - & - & - & $\cdot$ & $\cdot$ & . & . & . & 6 & 6 & 1 & 1 \\
\hline & Unidentified & & - & - & 8 & 8 & - & . & 5 & 5 & - & - & 11 & 18 \\
\hline & Total & & 165 & 246 & 22 & 24 & 540 & 904 & 46 & 55 & 225 & 456 & 67 & 128 \\
\hline
\end{tabular}


stems reached $41.0 \%$. The species with over 20 live individuals were $A$. cf. spectabilis $(\mathrm{n}=52)$, P. effusa (39), P. schomburgkiana (29), H. burchellii (23), and these 4 species accounted for $63.6 \%$ of all individuals. The number of dead individuals was also high for $P$. effusa (22) and P. schomburgkiana (11).

Six species were found in all quadrats. Fourteen species were common to both Q1 and Q2, and 63 new species appeared in Q2. The number of species common to both Q2 and Q3 was 22 , and the number of new species in Q3 was 17. At the family level, 21 families appeared in Q1, 35 families in Q2 and 20 families in Q3. Seventeen families were common to Q1 and Q2, 20 families to Q2 and Q3, and 10 families appeared in all quadrats.

Although all three quadrats are in early stage of secondary forest succession, tree height increased and the forest started to develop strata along the sequence, which represents approximately 20 years of succession. All individuals were concentrated in the class $0-5 \mathrm{~m}$ in Q1, more classes were present in Q2 and the highest class reached $25-30 \mathrm{~m}$ in Q3 (Fig. 1.A.). In terms of $D^{2} H$, there was a peak in $10^{2}-10^{3}$ class in Q1, this peak moved to a larger class and the range became wider in Q2, finally shifted up to the $10^{4}-10^{5}$ class in Q3 (Fig. 1.B.). The difference between Q1 and Q3 is 1 to 2 orders of magnitude.

When comparing the total $\mathrm{D}^{2} \mathrm{H}$ per quadrat $\left(/ 400 \mathrm{~m}^{2}\right)$ by summing the $\mathrm{D}^{2} \mathrm{H}$ of each tree in the quadrat, Q1 had $0.64 \mathrm{~m}^{3} \mathrm{Q} 2$ had $12.07 \mathrm{~m}^{3}$ and Q3 had $16.72 \mathrm{~m}^{3}$ (in Q1, the total $\mathrm{D}^{2} \mathrm{H}$ (/
$48 \mathrm{~m}^{2}$ ) was converted into the value per $400 \mathrm{~m}^{2}$ ). Assuming the value at the clear cutting to be zero, the growth curve is approximately synodal (Fig. 2 ), since total $D^{2} H$ increased tremendously during the 7 years between Q1 and Q2, but slowed during the 9 years between Q2 and Q3.

\section{Differences of Vessel Parameters among Species}

Examples of stem cross sections are shown in Figure 3. Group A contains tree species with large vessels and Group B with small ones. $S$. paniculatum has the largest diameter among the 18 species $(0.158 \pm 0.036$ $\mathrm{mm}$ ) and the summation of each vessel area accounts for $7.4 \%$ of the entire image investigated. One or a few united vessels are randomly distributed (diffused arrangement). The axial parenchyma is vasicentric, one of the paratracheal parenchyma types which enclose vessels, but the ray parenchyma is not obvious. The diameter of B. nitida is as large as $S$. paniculatum $(0.152 \pm 0.046 \mathrm{~mm})$, and the total area is also large $(5.8 \%)$. The diameter is larger in the early wood than in the late wood, which is categorized as a semi-ring arrangement. Sometimes, a few vessels are united in a radial pattern, and the vasicentric parenchyma encloses the vessels. The ray parenchyma is clearly confirmed. Both the diameter and the area of $E$. schomburghii are slightly smaller than those of former examples, but are still large among the 18 species $(0.137 \pm 0.024 \mathrm{~mm}, 5.1 \%)$. The distribution pattern is a diffused. A few ves- 


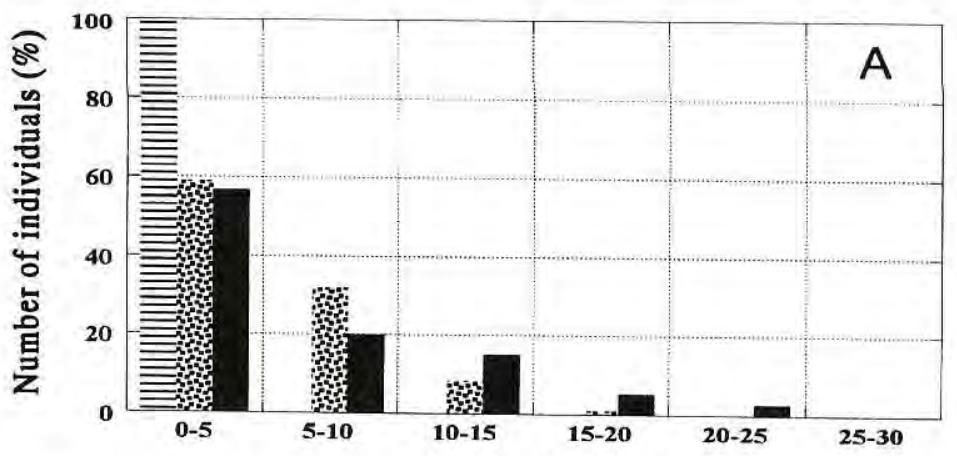

Height class (m)

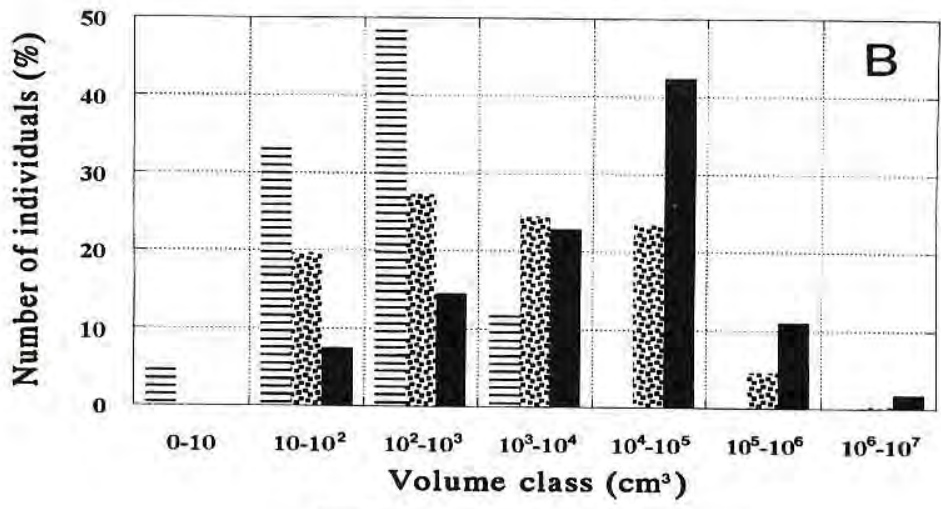

\begin{tabular}{lllll}
\hline$=$ & $\mathbf{Q 1}$ & $\mathbf{Q} 2$ & $\mathrm{Q3}$ \\
\hline
\end{tabular}

Figure 1. Comparison of forest structure across three secondary forest succession ages in the Caxiuanã National Forest, Pará, Brasil. A. tree height classes ( $\%$ live individuals). B. D ${ }^{2} \mathrm{H}$ classes ( $\%$ live individuals).

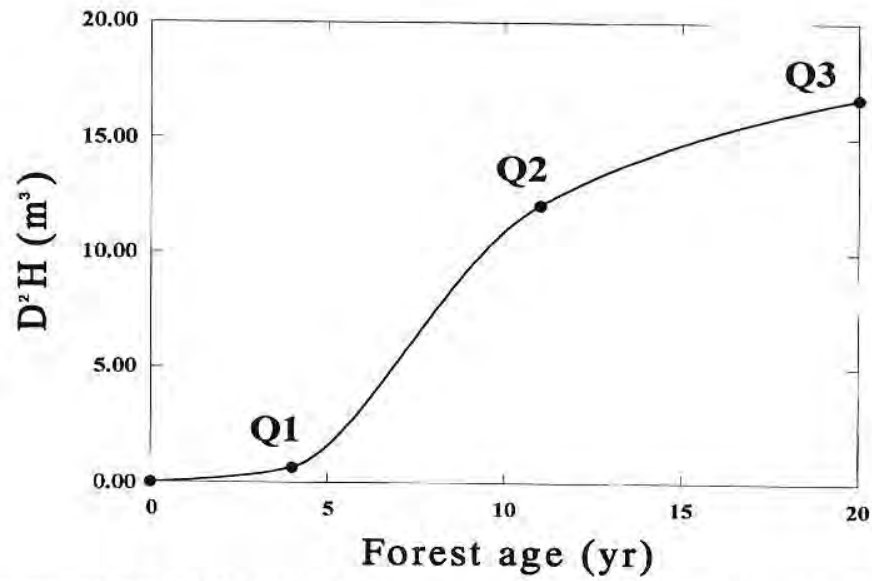

Figure 2. Increase of total $\mathrm{D}^{2} \mathrm{H}\left(/ 400 \mathrm{~m}^{2}\right)$ from clear cutting to the final secondary forest succession stage studied in the Caxiuanã National Forest, Pará, Brazil. 


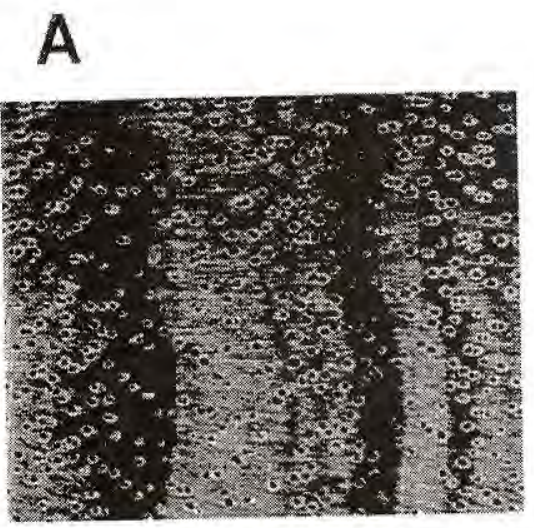

Sclerolobium paniculatum

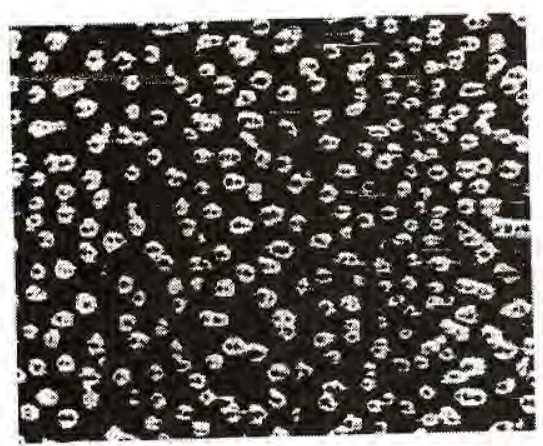

\section{Bowdichia nitida}

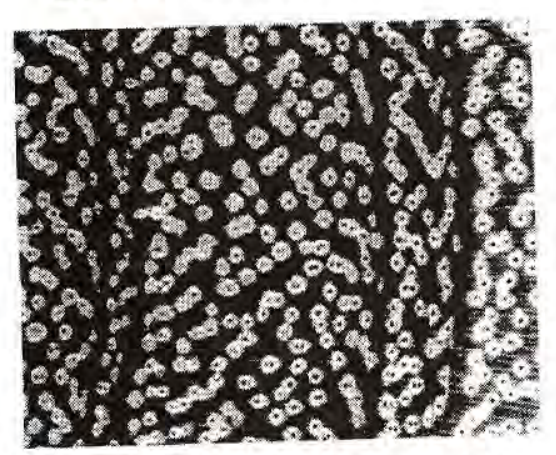

Enterolobium schomburghii

\section{B}

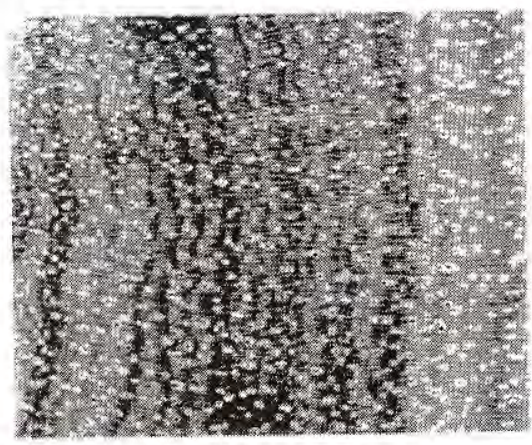

\section{Cupania rubiginosa}

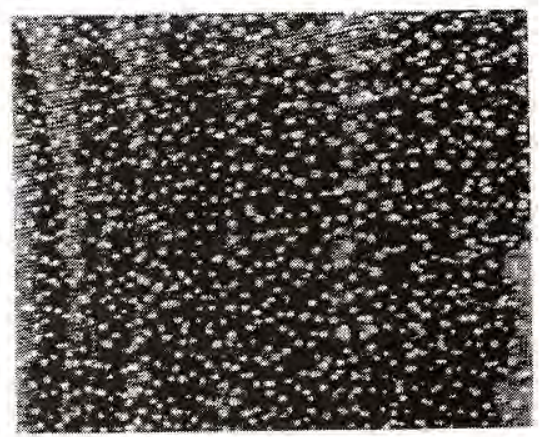

Cupania hispida

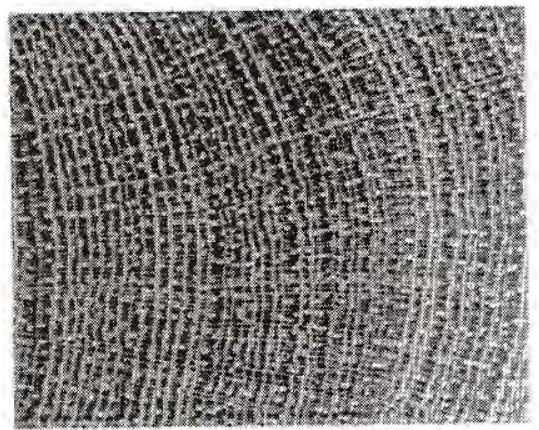

Poecilanthe effusa

Figure 3. Examples of vessels from stem cross sections obtained in 11-year old secondary forest (Q2) in the Caxiuanã National Forest, Pará, Brazil. 
sels are united to form clusters, and a couple of clusters are enclosed by the confluent parenchyma, but the ray parenchyma is hard to confirm.

On the other hand, tree species of Group B have small vessel diameters $(0.05-0.06 \mathrm{~mm})$, and the vessel area is also small (1.5-1.7\%). However, the number of vessels $\left(/ \mathrm{mm}^{2}\right)$ is higher, for example, 6.5 ( $C$. rubiginosa), 6.4 (C. hispida) and 8.3 (P. effusa). Because C. rubiginosa and C. hispida belong to the same family (Sapindaceae), they have a similar wood structure (diffused arrangement, vasicentric parenchyma). P. effusa also has a diffused arrangement, but the parenchyma is independent from the vessels. The apotracheal parenchyma is tangentially distributed. All the three species have narrow ray parenchyma.

Because the number of vessels decreases when vessel diameter increases, the relationship had a negative correlation coefficient $(\mathrm{r}=-0.88$; $\mathrm{p}<0.01$ ). Further, the relationship between vessel diameter and area had a positive correlation coefficient $(\mathrm{r}=0.90$; $\mathrm{p}<0.01)$ because vessel area was dependent on diameter. In addition, species of Group A have large diameter vessels but the number of vessels is small, while Group B has small diameter vessels and a large number of them.

\section{Tree Growth and Vessel Para- meters}

Both the area and diameter of the vessel parameters had a positive correlation coefficient to $\mathrm{D}^{2} \mathrm{H}$, but the number of vessels had a negative correlation coefficient (Fig. 4). The relationship between vessel area and $\mathrm{D}^{2} \mathrm{H}$ showed that the $\mathrm{D}^{2} \mathrm{H}$ of $\mathrm{PE}, \mathrm{CR}$ and $\mathrm{CH}$, whose area was smaller than $2 \%$, was 3,269 , 32,438 and $34,063 \mathrm{~cm}^{3}$ respectively. In contrast, the $\mathrm{D}^{2} \mathrm{H}$ of $\mathrm{ES}$, whose area was $5.1 \%$, was $639,812 \mathrm{~cm}^{3}$, and the $\mathrm{D}^{2} \mathrm{H}$ of BN (vessel area: $5.8 \%$ ) and SPN (vessel area: $7.4 \%$ ) was $1,062,500 \mathrm{~cm}^{3}$. The difference between PE and BN or SPN was about $325 \mathrm{x}$. In fact, the mean DBH of PE was $2.00 \pm 0.25 \mathrm{~cm}$, and the tree height was $4.20 \pm 0.75 \mathrm{~m}$, while they were $25.0 \mathrm{~cm}$ and $17.0 \mathrm{~m}$ in both BN and SPN. The vessel diameter had a similar relation with $\mathrm{D}^{2} \mathrm{H}$. There was a tendency for the species with large diameter vessels to have large $D^{2} H$. On the other hand, the number of vessels was negatively correlated to $\mathrm{D}^{2} \mathrm{H}$, although the relationship was less clear $\left(r^{2}=0.31\right.$; $\mathrm{p}<0.05)$. The number of vessels per unit area tended to decrease as the tree size becomes larger.

The 18 species were classified into 7 canopy species ( $>20 \mathrm{~m}, \mathrm{AA}), 7$ intermediate species ( 5 to $20 \mathrm{~m}, \mathrm{AM}$ ) and 4 understory $(<5 \mathrm{~m}, \mathrm{AB})$. The species of Group AA seem to form a cluster in every scatter diagram (Fig. 4). For example, they are located in the upper right in vessel area vs $\mathrm{D}^{2} \mathrm{H}$ and vessel diameter vs $\mathrm{D}^{2} \mathrm{H}$ (Fig. 4.A, B), and are located in lower right in the number of vessels vs $\mathrm{D}^{2} \mathrm{H}$ (Fig. 4.C).

In addition, the classification of 77 species in Q2 was $17 \mathrm{AB}, 32 \mathrm{AM}$, and $28 \mathrm{AA}$. When compared to Q1, the percentage of $\mathrm{AB}$ decreased to $22.1 \%$ from $45.5 \%$, while the AA increased to $36.3 \%$ from $9.0 \%$. Also, when classifying the 77 species by the number of individuals, the percentage 

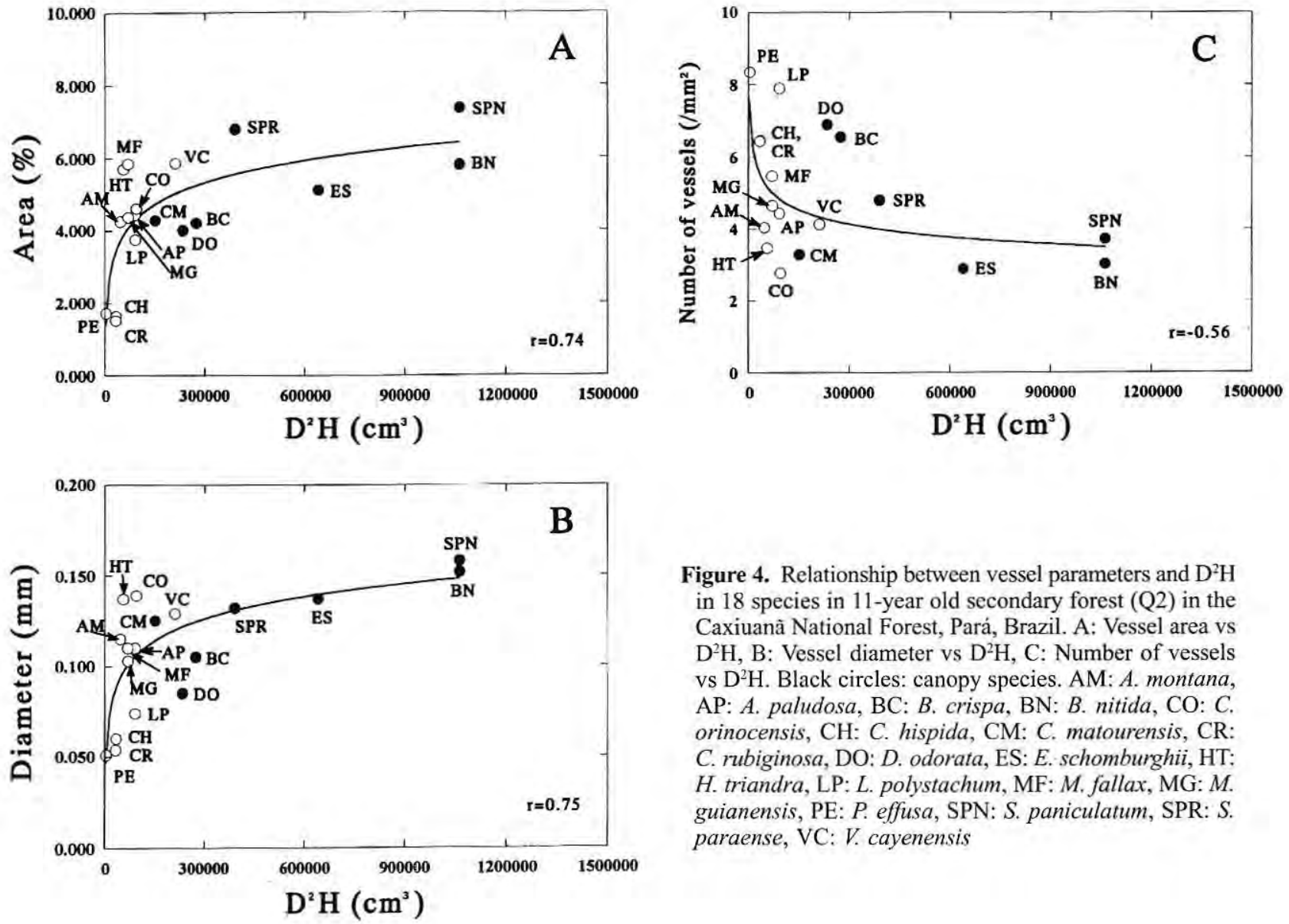

Figure 4. Relationship between vessel parameters and $D^{2} \mathrm{H}$ in 18 species in 11-year old secondary forest (Q2) in the Caxiuanã National Forest, Pará, Brazil. A: Vessel area vs $\mathrm{D}^{2} \mathrm{H}, \mathrm{B}$ : Vessel diameter vs $\mathrm{D}^{2} \mathrm{H}, \mathrm{C}$ : Number of vessels vs $\mathrm{D}^{2} \mathrm{H}$. Black circles: canopy species. AM: $A$. montana, AP: A. paludosa, BC: B. crispa, BN: B. nitida, CO: $C$. orinocensis, $\mathrm{CH}$ : C. hispida, CM: $C$. matourensis, $\mathrm{CR}$ : C. rubiginosa, DO: D. odorata, ES: E. schomburghii, HT: H. triandra, LP: L. polystachum, MF: M. fallax, MG: $M$. guianensis, PE: P. effusa, SPN: $S$. paniculatum, SPR: $S$. paraense, VC: V. cayenensis 
was 55.4 AB, 26.9 AM, and 17.8 AA, and the percentage of AA was 7 times that of Q1.

When investigating the quadrat of 12 species (AB: $5, A M: 7)$ with more than 10 individuals among $30 \mathrm{AB}$ and 49 AM species, most individuals of $P$. effusa (AB), C. hispida (AM), $C$. rubiginosa (AM) and $H$. burchellii (AM) appeared in Q2 and Q3. Because this strata does not develop soon after clear cutting, as in Q1, these four species are thought to be representative of $\mathrm{AB}$ and $\mathrm{AM}$. It was found that $P$. effusa had a high percentage of individuals with multiple stems, a large number of stems, but that mortality was also higher and $\mathrm{D}^{2} \mathrm{H}$ was smaller (Tab. 2). In Q3, the percentage of individuals with multiple stems increased to $60.7 \%$, the mean number of stems increased to $3.5 \pm 3.9$, the mortality also increased to $36.1 \%$. Hence, it is suggested that $P$. effusa has a growth strategy to have a large number of individuals and maintain itself by sprouting new stems, although the mortality is high because the species selects the forest floor as its habitat.

\section{Discussion}

Almeida et al. (1993), who car- ried out forest inventories in Caxiuanã for trees of $\mathrm{DBH}>10 \mathrm{~cm}$, recorded 338 species ( 50 families) in 4 ha of natural forest. Further, he reported that the species richness of other natural forest in Amazonia was 59 species, 29 families in 0.50 ha and 101 species, 30 families in 1.00 ha in Altamira, 121 species, 39 families in 1.00 ha in Capitão, 173 species, 38 families in 2.00 ha in Mocambo, 265 species, 39 families in 3.00 ha in Rio Xingu and 179 species, 47 families in 3.50 ha in Castanhal. We found that the species richness of Caxiuanã is extremely high, since our result showed 110 species, 39 families in 0.085 ha, approximately equivalent to a natural forest of 1 ha. However, the only common family between natural and secondary forests in Caxiuanã was Chrysobalanaceae. Also, according to the species list made by Lisboa et al. (1997), no species existed in common between these forest types at Caxiuanã. This indicates that the species composition of secondary forest is totally different from natural forests, and future plant succession will change the composition.

The fact that species have dis-

Table 2. Comparison of Cupania hispida SW, Cupania rubiginosa (Poir.) Radlk. and Poecilanthe effusa (Huber) Ducke in terms of percentage of individuals with multiple stems, number of stems per individual, percentage of dead individuals, and mean $D^{2} \mathrm{H}$ in 11-year old secondary forest (Q2) in the Caxiuanã National Forest, Pará, Brasil.

\begin{tabular}{lccc}
\hline & Cupania hispida & Cupania rubiginosa & Poecilanthe effusa \\
\hline Individual with mutiple stems (\%) & 35.7 & 29.9 & 48.6 \\
Number of stems per individual & $1.7 \pm 1.1$ & $1.5 \pm 0.8$ & $2.4 \pm 2.0$ \\
Dead individuals (\%) & 6.9 & 6.1 & 11.6 \\
$\mathrm{D}^{2} \mathrm{H}$ per individual $\left(\mathrm{cm}^{3}\right.$ ) & $8.951 \pm 13.072$ & $6.342 \pm 12.664$ & $3,269 \pm 5.090$ \\
\hline
\end{tabular}


tinct life strategies and have different vessel parameters is related to the difference of tree size. If a tree reaches the canopy layer, the height is favorable for photosynthesis, but it is necessary to pump a huge amount of water and the plant needs large sized vessels. This fact is explained by Poiseuille's law, that states that the amount of fluid which passes through a cylinder is proportional to the summation of fourth power of its diameter (Zimmermann, 1983). As tree size increases, the water potential gradient in stems and branches also increases (Calkin et al., 1986, Tyree \& Ewers, 1991). The tree copes with the gradient by increasing vessel diameter and area. Even if the tree has a large number of vessels, vessel area does not in- crease because mean diameter becomes smaller. In contrast, small sized species remaining near the forest floor do not need to transport water in large quantities. It is believed that the difference of vessel diameter and area is caused by this difference of height growth.

According to SUDAM/DRN (1981), who analyzed stem transections of 54 useful species at Curuá-una Forest Reserve near Santarem, Pará, and described their wood characteristics, tall species have large sized vessels (Fig. 5). Although individual tree size was unknown, 52 species were classified to $\mathrm{AA}$ and AM, following Rodrigues (1989) and Loureiro et al. (1997). The figure indicated that as vessel diameter increases and the number

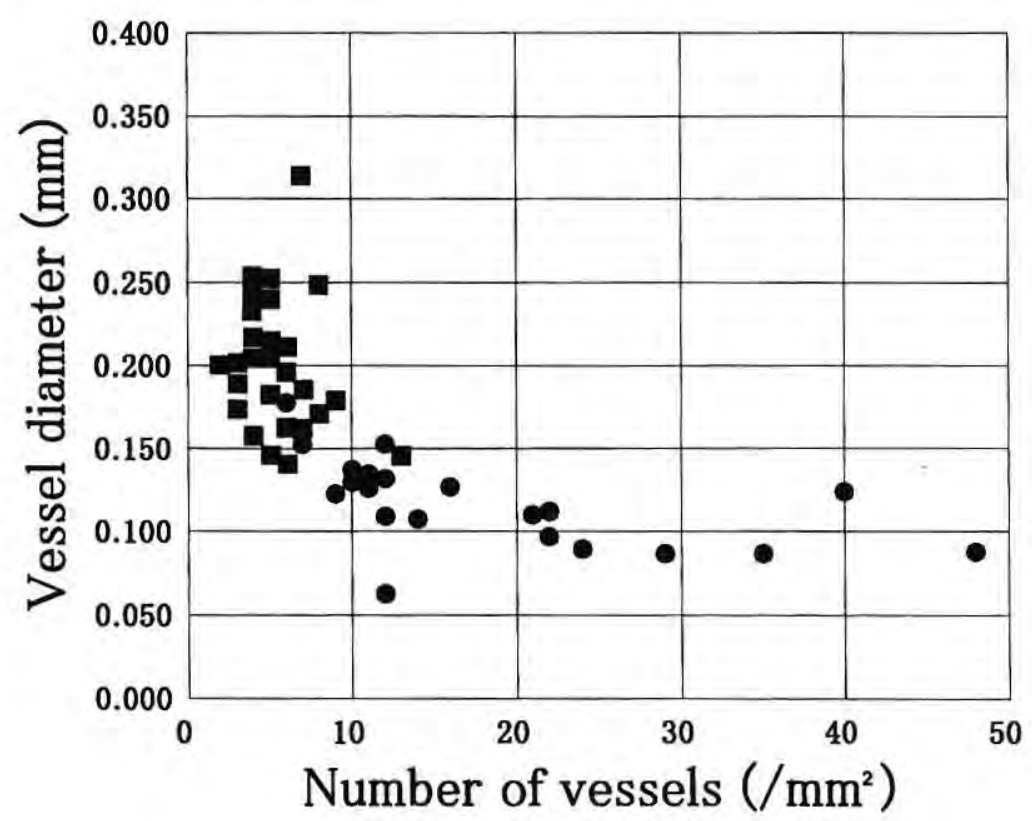

Figure 5. Relationship between vessel diameter and number of vessels of 52 species (black squares: AA, black circles: AM) in the Curuá-una Forest Reserve. Data were derived from SUDAM/DRN (1981). 
of vessels decreases, the share of AA becomes higher. This result is from a natural forest, and the species are limited to Groups AA and AM. However, the relationship between vessel number and diameter of our study seems to correspond to the upper left of the figure.

In this study, vessel parameters were compared to $\mathrm{D}^{2} \mathrm{H}$ and experimentally classified potential tree height, using stem transections obtained from juvenile secondary forests. The relationship of older secondary forests and natural forests in which the strata development progressed further has not yet been examined. It is necessary to investigate older forests to verify the results of this study. Further, species taken up in this study were diffused and semiring arrangements. This kind of study has not yet been conducted for ring and radial porous wood species, although most of tropical hardwood species have diffused porous wood (IAWA, 1989).

\section{Acknowledgements}

This study was supported by the 95' Overseas Scientific Expedition of the Japanese Ministry of Education, Science and Culture entitled "Survival strategy of tree species in the semi-arid and humid tropics of Brazil (Leader: Toshie Nishizawa)". We wish to express special thanks to local workers for their assistance in our fieldwork.

\section{Literature cited}

Almeida S.S.; Lisboa, P.B.; Silva, A.S.L. 1993. Diversidade floristica de uma comunidade arborea na estação científica "Ferreira Penna" em Caxiuanã (Pará). Boletim do Museu Paraense Emilio Goeldi, Série Botânica, 9(1):93-128.

Calkin, H.W.; Gibson, A.C.; Nobel, P.S. 1986. Biophysical model of xylem conductance in tracheids of the Fern Pteris vittata. Journal of Experimental Botany, 37:1054-1064.

Carvalho, J.O.P.; Silva, J.N.M.; Lopes, J. do C.A.; Montagner, L.H.; Carvalho, M.S.P. 1986. Composição florística de uma mata secundária no planalto de Belterra no Pará. In: Anais do $1^{o}$ Simpósio do Trópico Úmido, Vol. II, Flora e floresta. EMBRAPA/CPATU, Belém. pp.197-205.

De La Penha, G.M.; Lisboa, P.B.; Almeida, S.S. 1990. A estação científico do Museu Emilio Goeldi. Ciência em Museus, 2:87-96.

Fearnside, P.M. 1990. The rate and extent of deforestation in Brazilian Amazonia. Environmental Conservation, 17(3):213-226.

Fearnside, P.M. 1991. Desmatamento e desenvolvimento agrícola na Amazônia. In: Léna, P; Oliveira, E. (eds.) Amazônia, a fronteira agricola 20 anos depois, Museu Paraense Emílio Goeldi, Belém. pp. 207-222.

Federov, A.A. 1966. The structure of the tropical rain forest and speciation in the humid tropics. Journal of Ecology, 54(1):1-12.

Gatto, L.C.S. 1991. Relevo. In: IBGE. Geografia do Brasil, Vol.3, Região Norte. IBGE, Rio de Janeiro, pp.47-60.

IAWA 1989. IAWA list of microscopic features for hardwood identification. IAWA Bulletin 10(3):219-332.

Lisboa, P.B.; Silva; A.S.L., Almeida, S.S. 1997. Florística e estrutura dos ambientes. In: Lisboa, P.B. (ed.) Caxiuanã. Museu Paraense Emilio Goeldi, Belém. pp.163-183.

Loureiro, A.A.; De Freitas, J.A.; De Freitas, C.A.A. 1997. Essências madeireiras da Amazônia, Vol.3. INPA, Manaus, 103p.

Platt, W.J.; Strong, D.R. 1989. Special feature - Tree fall gaps and forest dynamics. Ecology, 70(3):535-576.

Rodrigues, R.M. 1989. Flora (nome, 
classificação cientifica, e descrição detalhada dos arbustos, plantas, etc.). In: Rodrigues, R.M. (ed.) A flora da Amazônia. CEJUP, Belém. pp.23-205.

Schweingrüber, F.H. 1988. Tree-ring growth and the site. In: Schweingrüber, F.H. (ed.) Tree-rings. Basics and applications in dendrochronology. Kluwer Academic Publishers, Dordrecht. pp.95-142.

Shimaji, K.; Sudo, S.; Harada, H. 1976. Cell structure of broadleaf trees. In: Shimaji, K., Sudo, S., Harada, H. (eds.) Wood anatomy. Morikita Shuppan, Tokyo. pp.129-187. (in Japanese)

SUDAM/DRN 1981. Madeiras da reserva florestal de Curuá-Una, estado do Pará: caracterização anatômica, propriedades gerais e aplicações. SUDAM, Belém. 118p.

SUDAM/PHCA 1984. Atlas climatológico da Amazônia. SUDAM, Belém. 125p.

Taylor, C.M.; Silander, S.; Waide, R.B.; Pfeiffer, W.J. 1995. Recovery of a tropical forest after gamma irradiation: a 23year chronicle. In: Lugo, A.E.; Lowe, C. (eds.) Tropical forests: management and ecology, Ecological Studies 112. Springer Verlag, New York. pp.258-285.

Tyree, M.T.; Ewers, F.W. 1991. The hydraulic architecture of trees and other woody plants. New Phytologist, 119:345-360.

Vazques-Yanes, C.; Orozco-Segovia, A. 1994. Signals for seeds to sense and respond to gaps. In: Caldwell, M.M.; Pearcy, R.W. (eds.) Exploitation of environmental heterogeneity by plants. Academic Press, San Diego. pp.209-236.

Zimmermann, M.H. 1983. The HagenPoiseuille equation and its implications. In: Zimmermann, M.H. (ed.) Xylem structure and the ascent of sap. Springer Verlag, New York. pp.13-15.

Aceito para publicação em 26/03/2002 